\title{
Optimized Video Streaming over 802.11 by Cross-Layer Signaling
}

\author{
Ivaylo Haratcherev, Jacco Taal, Koen Langendoen, Reginald Lagendijk, and Henk Sips, \\ Delft University of Technology
}

\begin{abstract}
Seamless video streaming over wireless links imposes strong demands on video codecs and the underlying network. It is not sufficient that only the video codec or only the radio adapts to changes in the wireless link quality; efforts should be applied in both layers, and - if possible — synchronized. Also, the disturbing effect of possible background traffic over the same shared medium has to be taken into account. In this article we present a communication architecture for video streaming over 802.11 that is capable of adapting to changes in the link quality and sharing of the wireless channel in various use scenarios. Experimental results show that substantial improvements in the quality of the video can be obtained by applying link adaptation and cross-layer signaling techniques.
\end{abstract}

\section{INTRODUCTION}

Using wireless links for video streaming over the Internet is something that is becoming more and more common today. This combination makes the demanding world of real-time multimedia (which does not tolerate dropouts, for example) meet the quite imperfect - and capricious dark universe of radio links. A lot of effort is required to team up these worlds such that the stringent packet delay, jitter, and loss requirements of multimedia applications can be met by unstable and unreliable radio links.

Wireless links introduce bottlenecks for a number of reasons. First, communication over a wireless channel is simply not able to achieve the same quality (throughput, error rate, etc.) as its wired counterpart, which reduces the quality of the multimedia content that can be delivered. Second, in a mobile environment the channel conditions can change rapidly due to changing distance between the stations (user mobility), Rayleigh fading, and interference. Since multimedia streaming applications must deliver their content in real time, they are very sensitive to jitter in packet delivery caused by retransmissions in the underlying transport protocols. Third, multimedia streaming may be done over a shared medium like 802.11 and interfere with other users who are, for instance, downloading files.
With today's 802.11 products, the fundamental problems of wireless communication are aggravated by poor handling of the limited and imperfect resources (scarce spectrum, noisy medium) available to the radio. In particular, current transport protocols and device drivers do not actively control the user-available parameters of the 802.11 medium access control (MAC) layer; they use default values instead.

In this article we present an architecture for adaptive video streaming over 802.11. We show by a number of experiments that the real-time video streaming quality of the total system can be drastically improved by applying link adaptation and cross-layer signaling techniques. Link adaptation is a technique to handle the effects of changes in channel conditions and is typically employed at the link (MAC) level [1-4]. Basically, link adaptation is the process of automatically adjusting a number of radio/MAC parameters so that optimal quality of packet transmission is achieved. Cross-layer signaling can be used to pass link quality information to a video encoder such that, for example, the degree of compression is changed and consequently the data rate $[5,6]$. This mechanism can also be used to pass changes in throughput estimations to the application layer in case of medium sharing.

This article is organized as follows. We give an outline of our communication architecture and the use scenarios it is intended to cover. We describe the basics of link adaptation and give an overview of the various approaches to automatic rate control in 802.11 . We also describe the use of cross-signaling techniques in video streaming with and without medium sharing.

\section{COMMUNICATION SYSTEM ARCHITECTURE}

In designing an adaptive communication system, it is important to know what the inherent problems in wireless communication links are and their severity. Then, given the constraints the application imposes, such as the performance quality variations it can handle, an appropriate adaptive control mechanism can be designed. 
This control mechanism should keep the quality variations within the specified bounds, but not to the extent that control becomes too complex.

Figure 1 depicts the architecture of our adaptive wireless communication system for video streaming. In this architecture both the radio/MAC and the video encoder are rateadjustable, meaning that the transmission rate of the radio/MAC and the video coding rate of the video encoder can be dynamically changed (radio rate and video rate control components, respectively). The video encoder talks with the radio/MAC through the UDP/IP component layer of the system.

The rate control components obtain their information from the channel state predictor and medium sharing predictor components. The channel state predictor produces channel state information (CSI) used for link adaptation by the rate controller driving the radio/MAC component (path 1). This CSI is also supplied to the video encoder through a function that maps raw radio throughput to effective throughput, accounting for headers and so on (path 2). With medium sharing, the real link throughput is calculated from the CSI by a medium sharing pre-

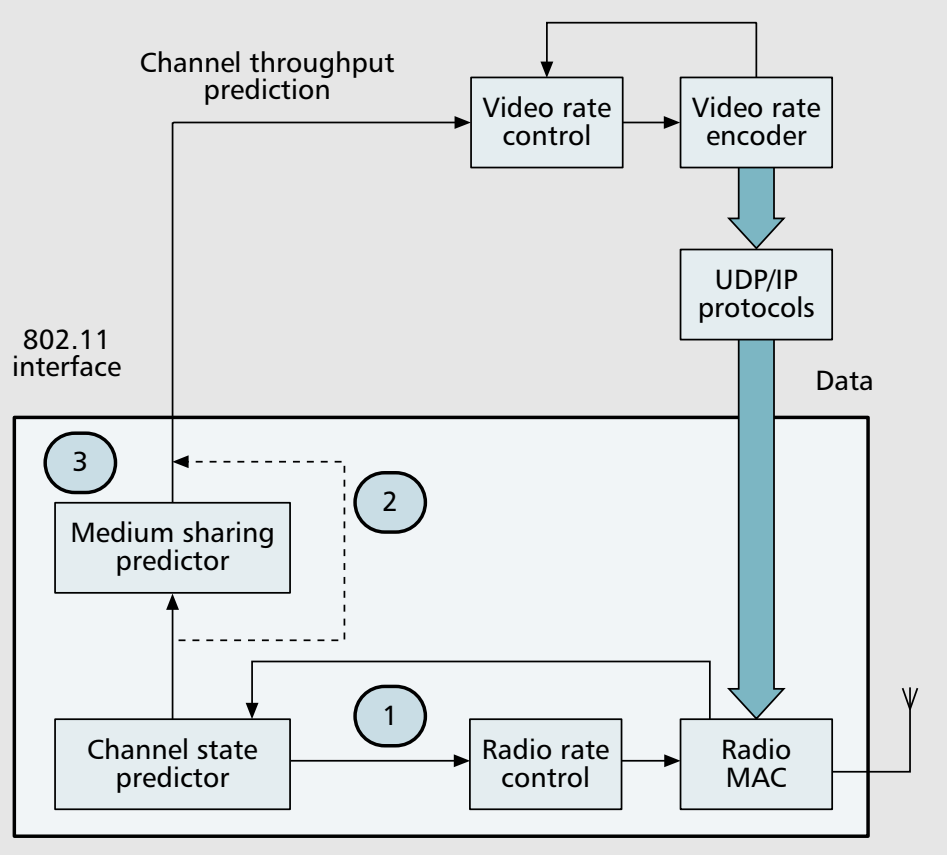

Figure 1. (1) Channel state prediction used for link adaptation; (2) channel throughput prediction without considering medium sharing; (3) channel throughput prediction considering medium sharing.

\begin{tabular}{ll|ll|l}
\multirow{2}{*}{ Medium } & \multicolumn{3}{c}{ Static channel } & \multicolumn{2}{c}{ Dynamic channel (movement) } \\
\cline { 2 - 5 } & $\begin{array}{l}\text { Low-quality } \\
\text { video }\end{array}$ & $\begin{array}{l}\text { High-quality } \\
\text { video }\end{array}$ & $\begin{array}{l}\text { Low-quality } \\
\text { video }\end{array}$ & $\begin{array}{l}\text { High-quality } \\
\text { video }\end{array}$ \\
\hline No sharing & - & - & 1 & $1+2$ \\
\hline Sharing & (3) & 3 & $1+3$ & $1+3$ \\
\hline
\end{tabular}

Table 1. Configuration for different video streaming scenarios; the numbers refer to the paths in Fig. 1. dictor (MSP, path 3), accounting for background traffic (and protocol overheads as before).

In Table 1 we show the communication system complexity as a function of a number of wireless use scenarios. First, consider the first row of the table (no medium sharing). In the first two entries we have scenarios where the channel is static (i.e., there is no movement or change in link quality). Here we do not need any channel state prediction and do not have to change any parameter in the radio. A typical example of such a scenario is a satellite link. The last two entries in the first row of the table depict scenarios that are dynamic. Here we definitely need channel state prediction to be able to adapt to the channel quality variations. Failing to do so will have catastrophic effects, no matter how smart the network layers above, just because a broken radio link means that no packets arrive at all.

Moreover, for high-quality video requirements, we also need the video encoder to have some information about the available throughput (paths $1+2$ ).

The picture changes completely when the wireless channel is shared (second row, Table 1). In [7] we have shown that the throughput can drop an order of magnitude, even with only a single additional user downloading. Therefore, we need to employ medium sharing prediction in addition to channel state prediction for all cases (static/dynamic channel, low/high video quality).

\section{LiNK AdAPTATION BASICS}

The IEEE 802.11 standard defines several MAClevel parameters (settings) that are available for tuning at the side of the wireless network interface card (NIC). The most important parameter available for adjustment is the transmit rate. In 802.11a, for example, the transmit rate can be set to $6,9,12,18,24,36,48$, and $54 \mathrm{Mb} / \mathrm{s}$. Each rate corresponds to a different modulation scheme with its own trade-off between data throughput and distance between the stations. This can be seen in Fig. 2; for clarity only the last four modulation schemes are shown.

The figure shows the performance in terms of the throughput for these modulation schemes vs. the signal-to-noise ratio (SNR). Note that distance is related to SNR as

$$
S N R \sim \frac{1}{\text { dist }^{\alpha}} .
$$

More complex modulation schemes like 64quadrature amplitude modulation (QAM) 3/4 offer larger throughput, but also have increased sensitivity to channel noise, and thus provide a shorter operating range. Usually, one wants to extend the operating range as much as possible and, at the same time, maximize the throughput. This can be done by proper (automatic) selection of the rate (modulation scheme) that gives the maximum throughput for certain conditions. Obtaining a reliable SNR measurement from the channel is difficult, and may require indirect solutions, as explained next. Knowing the optimal rate, we then compute the effective throughput at the user level, so the video codec can 
adjust its parameters to avoid overloading the communication link when channel conditions degrade. We use a simple model to convert the radio rate setting to available user space data throughput. Our model has been derived from the IEEE 802.11 standards [8] and is of the form

$$
T=\frac{8 R L}{8 L+b R+c},
$$

where $T$ is the throughput in megabits per second, $L$ is the length of a packet in bytes, $R$ is the data rate setting in megabits per second, and $b$ and $c$ are coefficients that depend on the 802.11 supplement. For 802.11a, $b=161.5$ and $c=156$. For $802.11 \mathrm{~b}, b=754$ in the case of a long preamble, or 562 in the case of short preamble, and $c=112$.

\section{Automatic MAC Rate Control}

The IEEE 802.11 standard [8] and its supplements do not specify any algorithm for automatic rate selection. To our knowledge, all of the known vendors of 802.11 equipment use statistics-based approaches to rate control, which are slow to respond to changes in link conditions. In the research community, another class of rate control algorithms has been studied. These control algorithms use SNR-related information as feedback to improve the sensitivity to changes in link conditions. We discuss both approaches below, as well as our hybrid solution that combines the advantages of statistics-based and SNR-based rate controllers.

\section{Statistics-Bbased Automatic RATE CONTROL}

An easy way to obtain the necessary information on link conditions is to maintain statistics about the transmitted data like the frame error rate (FER), acknowledged transmissions, and achieved throughput. Since these statistics are directly related to the effective user-level data throughput, they inherently guarantee that this throughput is maximized in the long term. Three basic types of statistics-based rate control can be distinguished: throughput-based, FER-based, and retry-based rate control. The throughput-based approach is the one that uses the most global type of statistic and is slowest. Retry-based control uses the most local statistic (number of retries per frame) and is fastest.

In throughput-based rate control a constant small fraction (10 percent) of the data is sent at two transmit rates adjacent to the current one (an adjacent rate is the next higher or lower one available). Then at the end of a specified decision window, the performance of all three rates is evaluated, and the rate with the highest throughput during the decision window is selected. To collect meaningful statistics, the decision window has to be quite large (i.e., about $1 \mathrm{~s}$ ); hence, the response to changes is rather slow.

In FER-based rate control, the FER of the data stream transmitted over the link is used to select an appropriate rate [9]. The FER can easily be determined since under 802.11 , all successfully received data frames are explicitly acknowledged by sending an ACK frame to the sender; hence, a missing ACK is a strong indica-

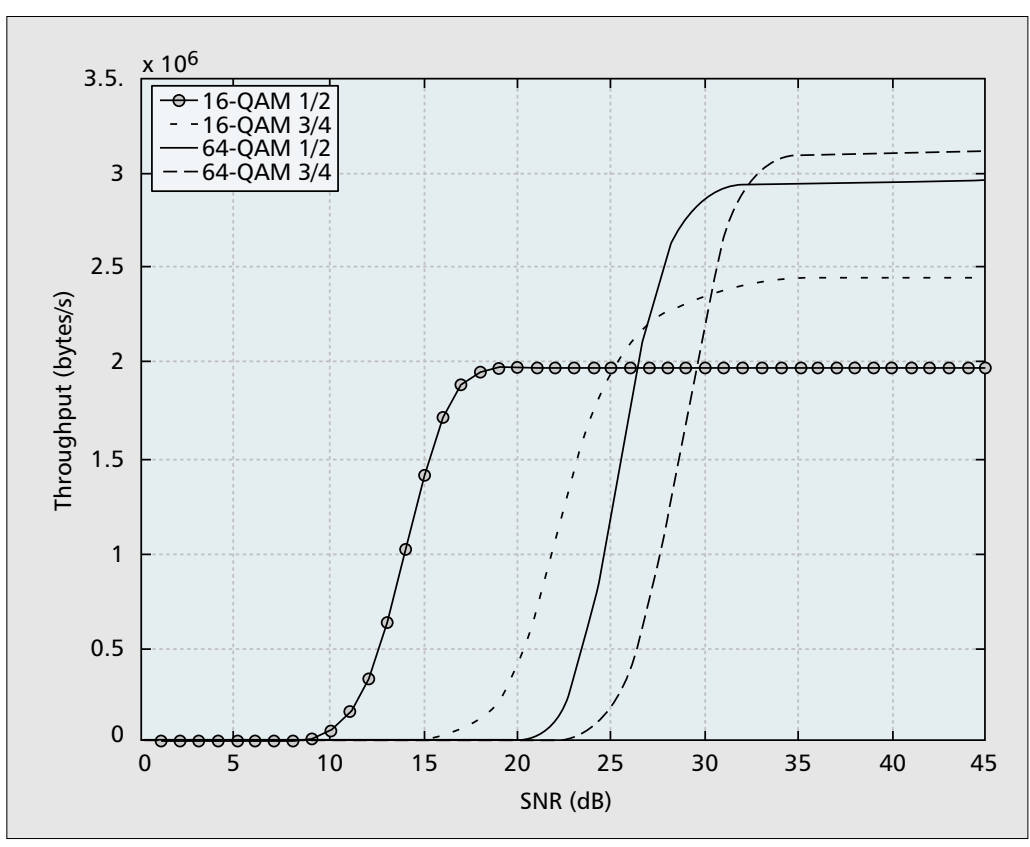

Figure 2. Throughput vs. SNR for some 802.11a modulation schemes.

tion of a lost data frame. By counting the number of received ACK frames and the number of transmitted data frames during a rather short time window, the FER can be computed as the ratio of the two. The width of the time window and rate switching thresholds are critical for the performance of the FER-based algorithm. The optimal settings of the parameters are dependent on the link and application, but are generally fixed at design time, limiting the effectiveness of FER-based rate controllers.

An improvement over the FER-based approach is to downscale immediately when the MAC is struggling to transmit a frame correctly over the link; that is, to select the next lower rate after a small number of unsuccessful retransmissions (usually 5-10) [3, 4]. This approach has to be implemented in hardware, since precise control of rate setting between retransmissions (of the same frame) is required. The advantage of this retry-based approach is that it combines a very short response time (a few frames) for handling deteriorating link conditions (downscaling) with low sensitivity to traffic rates. The price to be paid is that the control algorithm is rather pessimistic. Relatively short error bursts cause long drops in throughput because upscaling to higher rates takes much longer than downscaling due to the need to collect a meaningful FER and prevent oscillation.

\section{SNR-Based Automatic Rate Control}

A fundamental limit of indirect statistics-based feedback is that it classifies link conditions as either "good" or "bad." This binary information provides some notion about the direction in which to adapt the rate setting, but does not suffice to select the appropriate rate at once. This leads to a slow step-by-step accommodation to large changes in conditions, and introduces the risk of oscillation in stable conditions. A better approach is to use direct measurements of the link conditions.

The SNR is directly related to the bit error rate 


\section{We are investigating the possibility of introducing advanced estimation techniques, such as Kalman filtering, in the radio rate controller. Although this might improve the quality of our predictions, the benefit of such techniques should be carefully studied. In our case the advantage is doubt- ful since it comes at the price of a heavy computational load.}

(BER) in the link, and hence to the FER. Consequently, the SNR is linked to the packet delay and jitter, and throughput, and has the potential to provide rich feedback for automatic rate control [1]. Knowing the current SNR and the throughputvs-SNR curves for each rate setting (e.g., Fig. 2) would solve the rate selection problem instantly.

Despite the advantages, SNR-based rate control has not been applied in practice yet because of the following three problems:

1)In reality, for certain link conditions the relation between the optimal rate and SNR is highly variable. This is due to the imperfectness of the models describing the radio channel.

2)It is not trivial to obtain a reliable estimate of the SNR of a link. Many radio interfaces only provide an uncalibrated signal strength indication (SSI).

3)The rate controller, which is on the sending side, in fact needs the SNR observed at the receiving side.

Most work on using SNR information for automatic rate control is based on simulation and does not consider the practical difficulties of obtaining good SNR estimates. It concentrates on the way in which the noisy and drifting SNR (problem 1) can be used to determine the correct rate setting [1, 10]. Holland et al. [11] do address the issue of how to communicate back SNR values (problem 3), but their rate selection algorithm still relies on a straight SNR threshold technique. Another approach is discussed in [2], where the assumption is made that the channel is symmetric, meaning that the SNR observed at either station is very similar for any given point in time. This assumption allows Pavon et al. to use the SNR of the last ACK frame as an indicator of the SNR at the other side, and to use it for selecting the rate of the next data frame to be sent.

\section{Hybrid Automatic Rate Control}

Both the statistics-based and SNR-based approaches have advantages and disadvantages. The statistics-based approach gives robust performance and inherently maximizes the throughput in the long term. However, the main drawback is its slow response to changing link conditions, which can be a source of problems for real-time applications. SNR-based rate control can respond very fast, but due to the uncertain and fluctuating relation between SNR information and BER of the link, it lacks stability and reliability. Therefore, a logical step forward is to combine the two approaches in a hybrid algorithm that provides both robustness and fast response.

We have implemented such SNR-based hybrid rate control. The core of this hybrid algorithm is a traditional statistics-based (throughput-based) controller. The decisions of the core controller can be overridden by a second feedback loop. This loop bounds the acceptable range of the signal strength indication of the acknowledged frames (SSIA) values for each rate, based on the specific knee in the throughput vs. SNR curve (Fig. 2). The SSIA is used instead of the SNR, since most radio interfaces provide only uncalibrated SSI information. To account for the drift and lack of calibration of the SSIA readings, we employ an adaptive adjustment logic that updates the values according to the recent history of channel conditions. For more details see [12].

Our hybrid rate controller should not be affected by collisions caused by transmissions from other stations. First, the throughput-based controller bases its decisions on overall throughput measured for adjacent rate settings over relatively long periods of time. So collisions will affect all throughputs simultaneously; hence, the relationship between them will not change, and consequently it will not change the controller decisions. Second, if there is a collision, there will be no ACK, so no SSIA will be returned by the radio chipset to the driver. Thus, the state of the second feedback loop of the rate controller will not change, and consequently the final decisions of the rate controller will also not be affected by collisions.

We are investigating the possibility of implementing advanced estimation techniques, such as Kalman filtering, in the radio rate controller as well. Although this might improve the quality of our predictions (of both SSIA values and throughput), the benefit of such techniques should be carefully studied. In our case the advantage is doubtful since it comes at the price of a heavy computational load. We perform predictions at the driver/firmware level; therefore, such a computational burden is probably unacceptable.

We have compared the decoded video quality for different rate control algorithms in an experiment. In this experiment we used two laptops, one of which was in a fixed position on a desk. The other laptop was carried out of the room, moved a few times up and down through the hallway, and then returned to the initial position next to the other laptop. By walking up and down the hallway we ensured that the conditions continuously improved or deteriorated, so we could inspect the behavior of the various rate control algorithms under different circumstances. During the whole experiment the first laptop streamed an H.263 encoded video of the "carphone" sequence in QCIF format to the second laptop, which decoded and recorded the received video.

A classical and easy measure for image quality is peak SNR (PSNR). The downside of this measure is that it does not necessarily correspond to human perception, and is not well suited to moving pictures where frames are sometimes missing. To overcome this shortcoming we employed two measures:

- Average PSNR: The average of the PSNRs of all frames

- Human perceptual quality

To obtain a perceptual measurement we showed each received video to 14 people who had to give a mark between 0 and $5(0=$ bad, $5=$ good). Both measures together should give a good indication of the effects of losing packets on the quality of the video.

We compared the performance of the following three algorithms: perfect (a fictitious algorithm with no lost packets), hybrid-based, and statistics-based. Table 2 shows for each algorithm the packet loss ratio, perceptual quality, and average PSNR.

The results in Table 2 show that the statisticsbased algorithm loses over 45 times more packets than the hybrid algorithm. The video quality 
for the hybrid algorithm is therefore $7.26 \mathrm{~dB}$ higher than for the statistics-based algorithm. The perceptual rating confirms this difference. As can be observed from the perceptual measurements, even low packet loss ratios give significantly lower qualities. This is due to the propagation of errors in consecutive frames. A typical effect of these errors is shown in Fig. 3.

\section{Cross-Layer Signaling}

At the application layer, the video encoder can also adapt to the link quality by, for example, changing the compression degree and thus modifying the data rate. This adaptation requires that the video encoder be able to sense the link quality (e.g., by getting feedback information from the decoder side). However, such a scheme is ineffective when the round-trip delays are too long. Using this approach also introduces additional overhead.

Our solution is use an adaptive video encoder on top of the hybrid rate control algorithm [13]. The video encoder will adapt based on the CSI provided by the channel state predictor (Fig. 1) and an additional forecast about what the link conditions are going to be in the next couple of tens of milliseconds.

The video codec we used is an H.263 codec modified to support interaction with the link layer. Our version of the H.263 encoder supports a video rate control algorithm (VRCA) that tries to achieve a certain rate by adjusting the quantization step size. The quantization step size is the main parameter that controls the compression of the video. This VRCA has been designed for constant bit rate (CBR) encoding, but can also be used to dynamically change the bit rate produced by the encoder. The resulting bit rate depends not only on the selected quantization step size, but also on the statistics of the picture itself. Therefore, the VRCA cannot set the bit rate beforehand and then expect that this bit rate will be exactly achieved.

The VRCA is implemented as a simple feedback control loop that consists of setting an initial quantization step size, encoding part of the picture, measuring the resulting intermediate bitrate, changing the step size accordingly and then continuing with the next part of the picture. In total there are nine parts of a picture frame for which the quantization step size can be adjusted, which generally is enough to be able to achieve a certain preset rate. With this algorithm, we are able to change the target bit rate for each individual frame, meaning that we have a maximum delay of $40 \mathrm{~ms}$ ( $25 \mathrm{frames} / \mathrm{s})$ to respond to changes in the channel.

In this way, we are able to constantly adapt the target video encoding rate for the VRCA according to information from the MAC rate control algorithm. By coupling the rate control algorithms of the MAC and the video coder in this manner, we can efficiently use the available transmission rate to maximize the picture quality.

The following experiment shows the effectiveness of our cross-layer signaling approach. One of the laptops was again placed in a fixed position, and the other followed a predetermined track. The track consisted of three parts: "lead-in,"

\begin{tabular}{llll} 
Algorithm & Packet loss & Perceptual quality & Average PSNR (dB) \\
\hline Perfect & $0.00 \%$ & 5.0 & 37.34 \\
\hline Hybrid & $0.15 \%$ & 3.0 & 36.59 \\
\hline Statistics-based & $7.01 \%$ & 0.4 & 29.33
\end{tabular}

Table 2. Packet loss, PSNR measurements, and perceptual quality rating for three different algorithms.

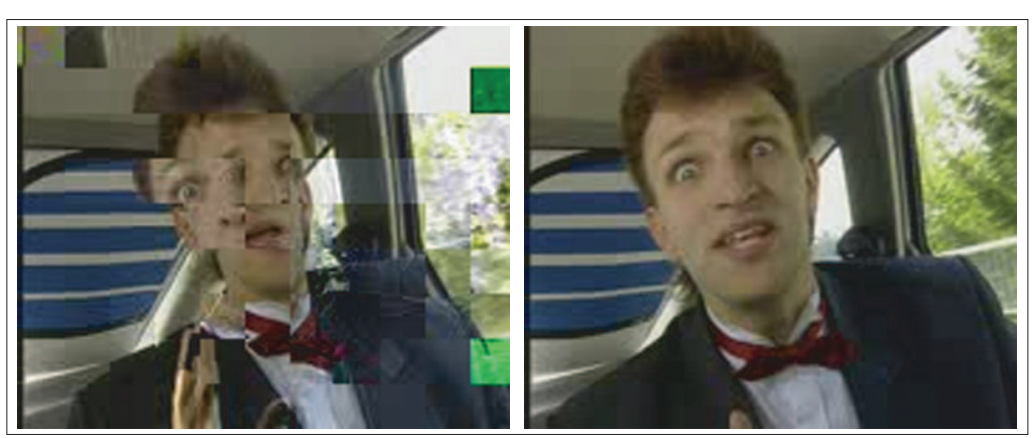

Figure 3. Standard rate control (left) and hybrid rate control (right).

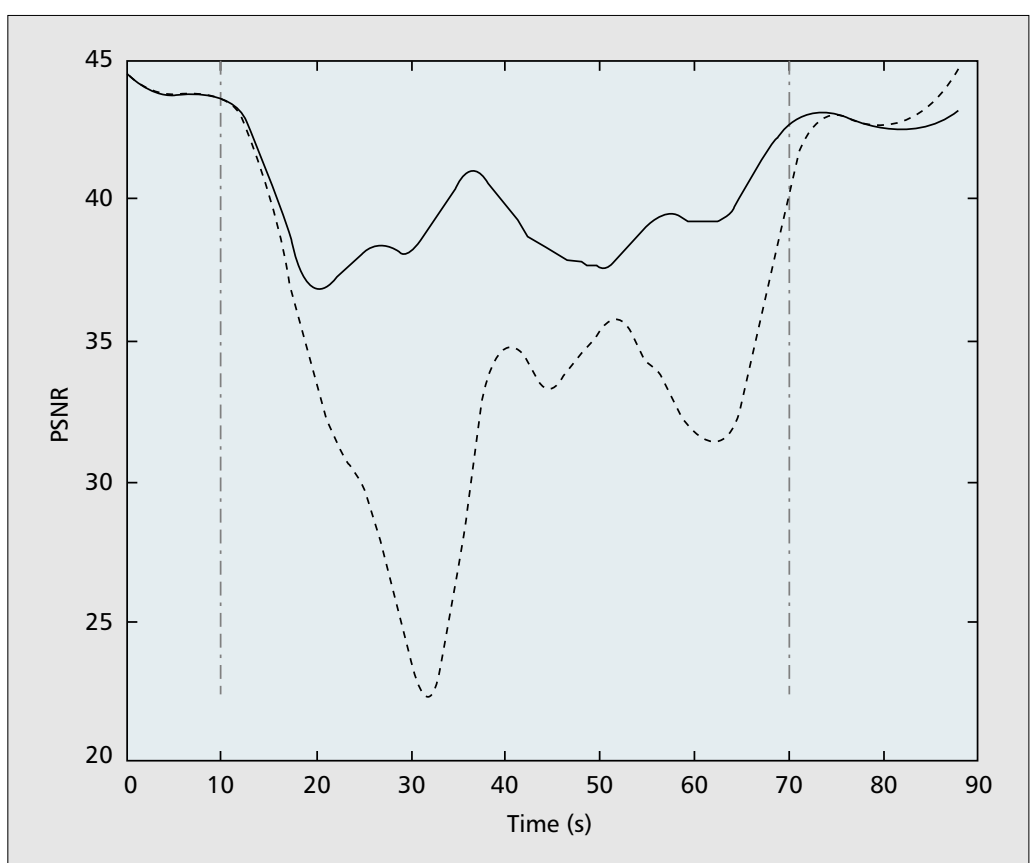

Figure 4. PSNR. The solid line shows how the quality (PSNR) changes during the experiment for cross-layer signaling. The dashed line shows the results for no cross-layer signaling.

which reached from the room to a specific start position in the hallway, waiting until a certain time elapses $(10 \mathrm{~s})$. Then the laptop was moved up and down the hallway three times (60 s). Finally, the laptop was placed back ("lead-out") in the room where the fixed laptop was $(20 \mathrm{~s})$.

We performed the above experiment for cross-signaling and no cross-signaling. For no cross-layer signaling, we set the target rate of the VRCA to the average rate obtained from the VRCA in the coupled case.

In Fig. 4 the quality (PSNR) is shown for the 
whole experiment ( $90 \mathrm{~s})$. On the left (0-10 s) the channel conditions are excellent; hence the high quality in both cases. The middle part is best described as having conditions changing from good to bad a few times. The right part has good conditions again.

As we can see in Fig. 4, cross-layer signaling yields a higher PSNR than no cross-signaling during the whole experiment. Table 3 summarizes the number of skipped frames (by the encoder), number of lost packets, and average PSNR in the period 10-70 s. Looking at the average PSNR in the $10-70$ s period, we conclude that the quality can be dramatically improved by informing the video codec of the actual present rate and a prediction for the near future.

\section{Medium Sharing Prediction}

The scenario described in the previous section assumes that the 802.11 medium is not shared by other users. If other users share the same medium, the throughput can drop significantly [7]. To be able to cope with this situation, we need a throughput predictor. Transmitting over the air is usually a bursty process (streaming, downloading), and the time for switching from an active to an inactive state is on the order of seconds. As a consequence, a throughput prediction could be based on the statistics of the observed throughput for each radio rate setting during previous transmissions. As the medium utilization changes slowly, this prediction should work fine for the period in which we are interested (a couple of tens of milliseconds), provided we have a way to detect when other users start using the radio channel.

\begin{tabular}{|llll}
\hline Case & $\begin{array}{l}\text { \# skipped } \\
\text { frames }\end{array}$ & \# lost packets & PSNR middle \\
\hline Cross-layer signaling & 50 & 13 & 39.3 \\
\hline No cross-layer signaling & 216 & 23 & 33.1 \\
\hline
\end{tabular}

Table 3. Cross-layer signaling and no signaling in rate control. The PSNR column shows the average PSNR over changing conditions in the period between 10 and $70 \mathrm{~s}$.

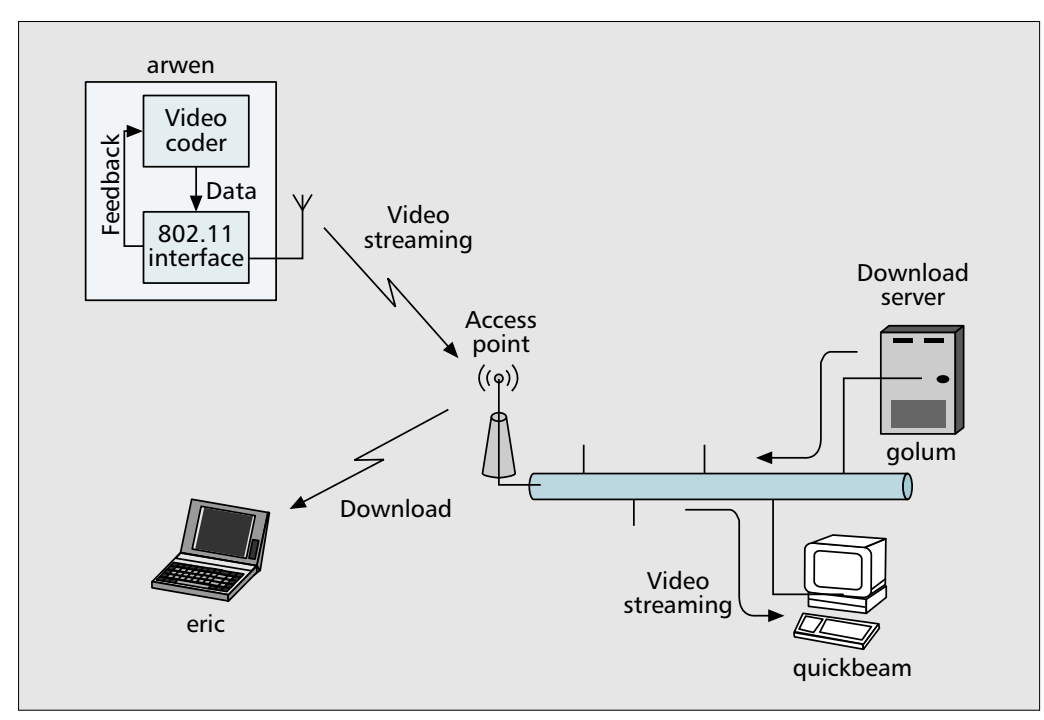

Figure 5. Our experimental setup.
To test the viability of having a good performance throughput estimator (the medium sharing predictor component in Fig. 1), we have implemented a throughput prediction emulator. This emulator is in fact a lookup table with throughput values for each rate setting, which we have measured beforehand in the presence of background traffic. The values are propagated as prediction values to the video encoder at the times we know the radio channel is used by other stations. In this way we can observe what the performance improvement would be in case of perfect throughput prediction.

To show this, we have done the following experiment. The setup consists of two desktops ("golum" and "quickbeam") on an Ethernet link, an 802.11a access point, and two laptops ("arwen" and "eric"), both running Linux (Fig. $5)$. The laptops are equipped with 802.11 a cards based on the Atheros AR5000 chipset, and the card driver uses the advanced hybrid rate control algorithm. The experiments were carried out by streaming a video file between arwen and quickbeam while "arwen" was moving, following a predetermined track. The track consisted of three parts: a lead-in of walking from the room where the access point lies to a specific start position in the hallway, then a move taking the laptop up and down the hallway for about $20 \mathrm{~s}$ (action); finally, the laptop was moved back (lead-out) into the room. While the video streaming took place, eric downloaded a file from golum (during the action part) for about $10 \mathrm{~s}$.

To evaluate the performance of the crosslayer signaling system in a shared medium (SM) scenario, we have examined two cases:

- No signaling. The video encoder has no indication of the actual throughput, and we set the target rate for the VRCA to a fixed value, which is the minimum throughput that would be obtained with no medium sharing (about $4 \mathrm{Mb} / \mathrm{s}$ ).

- SM signaling. The video encoder is informed about the effects of other users sharing the medium. The rate control loops of the MAC layer and video encoder are coupled through the throughput prediction feedback information provided by the MSP component emulator (Fig. 1)

Figure 6 shows the quality (PSNR) for the second (action) part of the experiment.

In the SM signaling case just a few frames were lost, since the video encoder properly reduced the video rate following the throughput prediction feedback. In the no signaling case, the higher video rate selected by the video encoder caused the wireless interface to choke during the download, and the video froze as a result of multiple frame losses. This "freeze" can be localized by regions with low PSNR. At about video frame 400 , the video data managed to get through again since the link conditions improved.

In periods when there is no background traffic (video frames from 100 to around 250, and from around 500 to 600 ), the PSNR of the SM signaling curve is slightly lower than that of the no signaling curve. This is because we used throughput prediction emulation for the duration of the experiment. This was to evaluate the loss of quality when there is pessimistic mispre- 
diction of the medium sharing (fewer stations with which to share the medium than predicted).

The mean PSNR over the whole measurement period of no signaling is $38.1 \mathrm{~dB}$, and for SM signaling it is $39.1 \mathrm{~dB}$. Focusing on the period with medium sharing (background download), we find that the mean PSNR is $28.3 \mathrm{~dB}$ for no signaling and $38.6 \mathrm{~dB}$ for $\mathrm{SM}$ signaling. In the SM signaling case a significant improvement (over $10 \mathrm{~dB}$ ) is achieved when the medium is shared.

\section{CONCLUSIONS AND FUtURE WORK}

In this article we have shown that cross-layer signaling in combination with advanced link layer MAC rate control has the potential of significantly improving the transmission quality of video streams over 802.11. As the size and scope of this article do not have enough room for more detailed analysis and experimental data, the interested reader is referred to [7, 12, 13].

Regarding medium sharing, we have only showed a proof of concept. Future work includes a real implementation of a medium sharing predictor instead of an emulator. We are also working on a generic application programming interface that can be used by 802.11 implementations to include cross-layer signaling and advanced algorithms for link adaptation.

\section{REFERENCES}

[1] K. Balachandran, S. R. Kadaba, and S. Nanda, “Channel Quality Estimation and Rate Adaptation for Cellular Mobile Radio," IEEE JSAC, vol. 17, no. 7, July 1999, pp. 1244-56.

[2] J. del Prado Pavon and S. Choi, "Link Adaptation Strategy for IEEE 802.11 WLAN via Received Signal Strength Measurement," IEEE ICC '03, vol. 2, Anchorage, AK, May 2003, pp. 1108-13.

[3] A. Kamerman and L. Monteban, "WaveLAN-II: A HighPerformance Wireless LAN for the Unlicensed Band," Bell Labs Tech. J., Summer 1997, pp. 118-33.

[4] A. J. van der Vegt, "Auto Rate Fallback Algorithm for the IEEE 802.11a Standard," Tech. rep., Utrecht Univ., 2002

[5] J.-C. Bolot and T. Turletti, "Experience with Control Mechanisms for Packet Video in the Internet," Comp. Commun. Rev., 1998.

[6] A. Ortega and M. Khansari, "Rate Control for Video Coding over Variable Bit Rate Channels with Applications to Wireless Transmission," Proc. 2nd IEEE Int'l. Conf. Image Processing, Oct. 1995.

[7] I. Haratcherev et al., "Link Adaptation and Cross-layer Signaling for Wireless Video-Streaming in a Shared Medium," WirelessCom 2005, Maui, HI, 2005.

[8] IEEE Std. 802.11, "Wireless LAN Medium Access Control (MAC) and Physical Layer (PHY) Specifications for 802.11a and 802.11b," 1999.

[9] B .E. Braswell and J. C. McEachen, "Modeling Data Rate Agility in the IEEE 802.11a WLAN Protocol," OPNETWORK 2001, Mar. 2001.

[10] T. Ue et al., "Symbol Rate and Modulation Level-Controlled Adaptive Modulation/TDMA/TDD System for High-Bit-Rate Wireless Data Transmission," IEEE Trans. Vehic. Tech., vol. 47, no., Nov. 19984, pp. 1134-47.

[11] G. Holland, N. H. Vaidya, and P. Bahl, "A Rate-Adaptive MAC Protocol for Multihop Wireless Networks," ACM MOBICOM '01, Rome, Italy, July 2001

[12] I. Haratcherev et al., "Hybrid Rate Control for IEEE 802.11," ACM Int'l. Wksp. Mobility Mgmt. and Wireless Access Protocols, Philadelphia, PA, Oct. 2004, pp. 10-18.

[13] I. Haratcherev et al., "Fast 802.11 Link Adaptation for Real-Time Video Streaming by Cross-Layer Signaling," Proc. Int'I. Symp. Circuits and Sys., Kobe, Japan, May 2005.

\section{BIOGRAPHIES}

IVAYLO HARATCHEREV (I.Haratcherev@ewi.tudelft.nl) is a Ph.D. student in the Parallel and Distributed Systems group at Delft University of Technology, The Netherlands. He

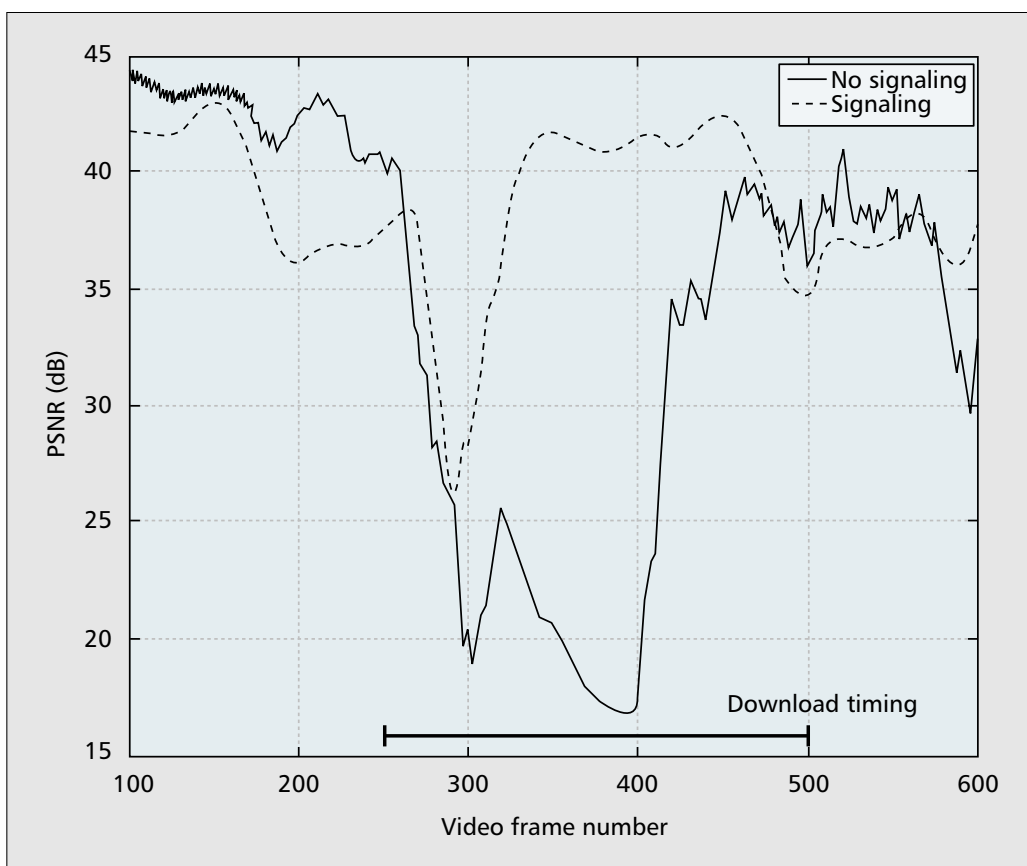

Figure 6. PSNR of the received video. The solid line shows the results for the no signaling case using a fixed rate of the video encoder. The dashed line shows the quality when SM signaling is on.

received his M.Sc. degree in systems and control engineering from the Technical University of Sofia, Bulgaria, in 2000. His research interests include automatic parameter control and QoS in wireless communications, embedded systems, and real-time control systems.

REGINALD L. LAGENDIJK (R.L.Lagendijk@ewi.tudelft.nl) received M.Sc and Ph.D. degrees in electrical engineering from Delft University of Technology in 1985 and 1990, respectively. He became an assistant professor at Delft University of Technology in 1987. He was a visiting scientist in the Electronic Image Processing Laboratories of Eastman Kodak Research, Rochester, New York, in 1991, and at Microsoft Research and Tsinghua University, Beijing, China, in 2000 and 2003. Since 1999 he has been a full professor in the Information and Communication Theory Group of the Technical University of Delft. At present his research interests include signal processing and communication theory, with emphasis on visual communications, compression, analysis, searching, and watermarking of image sequences. $\mathrm{He}$ is currently leading and actively involved in a number of projects in the field of intelligent information processing and data hiding for ad hoc and peer-to-peer multimedia communications.

KoEn LANGENDOEN (K.G.Langendoen@ewi.tudelft.nl) is an associate professor in the Parallel and Distributed Systems group at Delft University of Technology, The Netherlands. He earned an M.Sc. in computer science from the Vrije Universiteit, Amsterdam, in 1988 and a Ph.D. in computer science from the Universiteit van Amsterdam in 1993. His research interests include system software for parallel processing, wearable computing, embedded systems, and wireless sensor networks.

HENK J. SIPS (H.J.Sips@ewi.tudelft.nl) received an M.Sc degree in 1976 in electrical engineering and a Ph.D. degree in 1984 from Delft University of Technology. Currently he is a professorof computer science at Delft University of Technology. His research interests include parallel systems, distributed systems, mobile systems, and low-power systems.

JACCO R. TAAL (J.R.Taal@ewi.tudelft.nl) received his M.Sc degree in electrical engineering from Delft University of Technology in 2001. At present he is pursuing a Ph.D. degree at Delft University of Technology. His current research includes real-time video compression for wireless communications and multiple-description video streaming over peer-to-peer networks. 